Published in final edited form as:

Nat Immunol. 2017 July 19; 18(8): 826-831. doi:10.1038/ni.3790.

\title{
A guiding map for inflammation
}

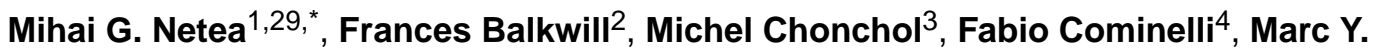
Donath $^{5}$, Evangelos J. Giamarellos-Bourboulis ${ }^{6}$, Douglas Golenbock ${ }^{7}$, Mark S. Gresnigt ${ }^{1}$, Michael T. Heneka ${ }^{8}$, Hal M. Hoffman ${ }^{9}$, Richard Hotchkiss ${ }^{10}$, Leo A.B. Joosten ${ }^{1,11}$, Daniel L. Kastner $^{12}$, Martin Korte ${ }^{13}$, Eicke Latz ${ }^{14,15}$, Peter Libby ${ }^{16}$, Thomas Mandrup-Poulsen ${ }^{17}$, Alberto Mantovani ${ }^{18}$, Kingston H. G. Mills ${ }^{19}$, Kristen L. Nowak ${ }^{3}$, Luke A. O'Neill ${ }^{19}$, Peter Pickkers $^{20}$, Tom van der Poll ${ }^{21}$, Paul M. Ridker ${ }^{22}$, Joost Schalkwijk ${ }^{23}$, David A. Schwartz ${ }^{24}$, Britta Siegmund ${ }^{25}$, Clifford J. Steer ${ }^{26}$, Herbert Tilg ${ }^{27}$, Jos W.M. van der Meer ${ }^{1}$, Frank L. van de Veerdonk ${ }^{1}$, and Charles A. Dinarello ${ }^{1,28}$
\end{abstract}

${ }^{1}$ Department of Internal Medicine and Radboud Center for Infectious Diseases, Radboud University Medical Center, Nijmegen, The Netherlands ${ }^{2}$ Barts Cancer Institute, Queen Mary University of London, Charterhouse Square, London, UK ${ }^{3}$ Division of Renal Diseases and Hypertension, University of Colorado, Denver, USA ${ }^{4}$ Digestive Health Research Institute, Case Western Reserve University, Cleveland, $\mathrm{OH}$, USA ${ }^{5} \mathrm{Clinic}$ of Endocrinology, Diabetes and Metabolism University Hospital and University of Basel, Switzerland ${ }^{6} 4$ th Department of Internal Medicine, National and Kapodistrian University of Athens, Medical School, Greece ${ }^{7}$ Division of Infectious Diseases and Immunology, University of Massacchussetts Medical School, Worchester, USA ${ }^{8}$ Department of Neurodegenerative Disease and Gerontopsychiatry/Neurology, University of Bonn, Bonn, Germany ${ }^{9}$ Division of Pediatric Allergy, Immunology, and Rheumatology, University of California at San Diego and Rady Children's Hospital of San Diego, USA ${ }^{10}$ Department of Anesthesiology, Medicine, and Surgery, Washington University School of Medicine, St Louis, MO, USA ${ }^{11}$ Department of Medical Genetics, luliu Haţieganu University of Medicine and Pharmacy, Cluj-Napoca Romania ${ }^{12}$ Inflammatory Disease Section, Metabolic, Cardiovascular and Inflammatory Disease Genomics Branch, National Human Genome Research Institute, US National Institutes of Health, Bethesda, Maryland, USA ${ }^{13}$ TU Braunschweig, Zoological Institute, Braunschweig, Germany and HZI, AG NIND, Braunschweig, Germany ${ }^{14}$ Institute of Innate Immunity, University Hospital Bonn, University of Bonn, Bonn, Germany ${ }^{15}$ Department of Infectious Diseases \& Immunology, UMass Medical School, Worcester, MA, USA ${ }^{16}$ Brigham and Women's Hospital, Harvard Medical School, Boston, MA, USA ${ }^{17}$ Department of Biomedical Sciences, University of Copenhagen, Copenhagen, Denmark ${ }^{18}$ Humanitas University and Humanitas Clinica Research Center, Rozzano, Milano, Italy ${ }^{19}$ School of Biochemistry and Immunology, Trinity Biomedical Sciences Institute, Trinity College Dublin, Ireland ${ }^{20}$ Department of Intensive Care Medicine, Radboud University Medical Center, Nijmegen, The Netherlands ${ }^{21}$ Center of Experimental and Molecular Medicine, Division of Infectious Diseases, Academic Medical Center, University of Amsterdam, Amsterdam, the Netherlands ${ }^{22}$ Center for Cardiovascular Disease Prevention, Brigham and Women's Hospital, Harvard Medical School, Boston, MA USA ${ }^{23}$ Department of Dermatology, Radboud University Medical Center, Nijmegen,

"Corresponding author: Mihai G. Netea, Department of Internal Medicine, Radboud University Medical Center, Geert Grooteplein 8 , 6525GA Nijmegen, The Netherlands, Tel: +31-24-3618819, mihai.netea@ radboudumc.nl. 
The Netherlands ${ }^{24}$ Division of Pulmonary Sciences and Critical Care Medicine, Department of Medicine, University of Colorado, Denver, USA ${ }^{25}$ Department of Medicine (Gastroenterology, Infectious Diseases, Rheumatology), Charité - Universitätsmedizin Berlin, Campus Benjamin Franklin, Berlin, Germany ${ }^{26}$ Departments of Medicine and Genetics, Cell Biology and Development, University of Minnesota Medical School, Minneapolis, Minnesota, USA ${ }^{27}$ Department of Internal Medicine I, Gastroenterology, Hepatology \& Endocrinology, Medical University Innsbruck, Austria ${ }^{28}$ Department of Medicine, University of Colorado Denver, Aurora, CO, USA ${ }^{29}$ Human Genomics Laboratory, Craiova University of Medicine and Pharmacy, Craiova, Romania

\section{Abstract}

Biologists, physicians and immunologists contributed to increasing the understanding of the cellular participants and biological pathways involved in inflammation. Here we provide a general guide map to the cellular and humoral contributors of inflammation, as well as the pathways that characterize it in specific organs and tissues.

Inflammation is viewed as the driving factor in many diseases, including atherosclerosis, cancer, autoimmunity and chronic infections 1 and a major contributor in age-related conditions2. The classical definition of inflammation comprising rubor(redness), calor (warmth), dolor (pain) and tumour (swelling), as described by Celsus (30BC-38AD) and functio laesa (loss of function), added by Galen (129AD-210AD), has persisted in modern times. Functionally, inflammation is broadly defined as a protective response of the organism to stimulation by invading pathogens or endogenous signals such as damaged cells, resulting in the elimination of the initial cause of injury, clearance of necrotic cells, and tissue repair. However, due to the complex and often simultaneous molecular, immunological and physiological processes involved in the inflammatory reaction, a clear definition of inflammation presents a challenge. Here we provide a guide of the sequence of events initiated during inflammation (Fig. 1), the main mechanisms leading to the resolution of inflammation (Fig. 2), and provide an overview of the most important characteristics of the inflammatory process in various tissues and diseases (Table 1).

\section{Basic elements of inflammation}

Inflammation is induced when host cells sense evolutionarily conserved structures on pathogens (PAMPs) or endogenous stress signals (DAMPs) through germline-encoded pattern recognition receptors (PRRs)3. PRRs are mainly expressed by myeloid cells such as monocytes, macrophages, neutrophils and dendritic cells, but also by lymphocytes, fibroblasts and epithelial cells4. Cellular stimulation triggers inflammatory processes through the release of proinflammatory cytokines and chemokines. Tumour necrosis factor (TNF) and interleukin-1 $\beta$ (IL-1 $\beta$ ) have autocrine and paracrine effects leading to the local activation of macrophages and neutrophils, but when released in large amounts can exert endocrine effects such as induction of acute phase proteins from the liver, activation of platelets, fever, fatigue and anorexia. Cytokines also activate endothelial cells to increase the vascular permeability and facilitate entrance of immune cells into the tissues at the site of 
infection, but can also lead to capillary leakage, vasodilation and hypotension 5, 6 (Fig. 1). The main function of chemokines is to recruit additional immune cells to the site of infection7, including neutrophils that exert a crucial role for the phagocytosis and killing of pathogens8,9. $\mathrm{T}_{\mathrm{H}} 1$-derived IFN- $\gamma$ activates neutrophils, while $\mathrm{T}_{\mathrm{H}} 17$ and innate lymphoid cells (ILC)-derived IL-22 acts on epithelial cells to stimulate production and release of antimicrobial peptides (AMPs), including defensins 10 .

In the bloodstream, activated monocytes and neutrophils release cytokines, which in turn stimulate the release of prostaglandins that mediate signs and symptoms of illness (somnolence, fatigue, fever) by acting on hypothalamus11. An important component of inflammation in the circulation is the activation of the complement system, which mediates microbial opsonisation and killing, and generates inflammatory peptides such as $\mathrm{C} 3 \mathrm{a}$ and C5a12.

\section{Basic elements of resolution}

Mechanisms that shut down the inflammatory response have paramount importance for the return to homeostasis (Fig. 2). Resolution is not simply the expression of elimination of the stressing agent, but an active process that involves functional reprogramming of involved cells with production of ad hoc mediators. Several mechanisms inhibit inflammation. IL-10 suppresses the production of proinflammatory cytokines 13 and derives mainly from regulatory T cells. IL-37, a member of the IL-1 family, broadly suppresses inflammation, as does TGF- $\beta$ released from monocytes and platelets14. Cleaved extracellular domains of cytokine receptors, such as soluble TNFR and IL-1R, serve as decoy receptors and limit inflammation by binding and neutralizing their respective cytokine. Receptor antagonists, such as the IL-1R antagonist (IL-1Ra), bind to IL-1R without inducing an intracellular signal and as such inhibiting the biological activity of IL-1 $a$ and IL-1 $\beta 15$. Complement inhibitors also modulate inflammation 16 and prostaglandins and lipid mediators such as resolvins exert negative feed-back loops by suppressing the transcription and release of cytokines. Acute phase proteins induced during inflammation, such as a-1 antitrypsin (AAT), have broad anti-inflammatory properties17. Additional anti-inflammatory mechanisms include stress hormones, in particular corticosteroids and catecholamines, negative regulators of TLR signaling such as IRAK-M and A20, and miRNAs, such as miR-146 or miR-125. Neuro-immuno-regulatory mechanisms (the so-called immune reflex) provides an anti-inflammatory negative feedback triggered by peripheral sensory input transmitted through the afferent vagus nerve to the brainstem, with subsequent activation of the efferent vagus and splenic nerve18, release of norepinephrine in the spleen and secretion of acetylcholine by a subset of $\mathrm{CD}^{+} \mathrm{T}$ cells, which inhibits proinflammatory cytokine production by macrophages. On the other hand, an anti-inflammatory response that is too pronounced or persistent may render the host vulnerable to secondary infections 19 .

Here we provide an up-to-date, informative guide to inflammation and summarizes the main features of acute or chronic inflammation in specific human diseases (Table 1). 


\section{Sepsis}

In sepsis,_the release of DAMPs or alarmins from injured host cells activates PRRs that also recognize PAMPs, giving rise to a vicious cycle of sustained hyper-inflammation20. Yet, an ineffective antimicrobial host defense often accompanies this condition. Proinflammatory cytokines produced following recognition of the invading pathogens by PRRs21 can help protect the host but also promote tissue injury during overwhelming sepsis. Unrestrained activation of complement contributes to organ failure, and C5a blockade improves the outcome of experimental sepsis. Local activation of the coagulation system in sepsis helps to confine pathogens to the primary site of infection, while systemic activation may result in disseminated intravascular coagulation, microvascular thrombosis and bleeding22(Table 1). Enhanced leukocytes and platelet adherence to the endothelial surface and transmigration results in vascular inflammation and disruption of endothelial cell barrier, causing leakage of intravascular proteins to the extravascular space, tissue oedema, and reduced microvascular perfusion. When severe, these abnormalities may lead to organ dysfunction and even death23.

\section{Acute intestinal inflammation}

Acute intestinal inflammation can protect by eliminating infectious, toxic and other injurious agents, while initiating the process of repair. Chronic inflammation in the gastro-intestinal tract results from either repeated acute injury and/or impaired resolution of inflammation, leading to conditions such as chronic gastritis and peptic ulcer disease, chronic pancreatitis, celiac disease and inflammatory bowel diseases. In Crohn's disease and ulcerative colitis, despite the unknown precise aetiology, treatment aims to dampen inflammation and its consequences24. Corticosteroids reduce inflammatory flares, but in most cases do not maintain remission. Immunosuppressants such as azathioprine and methotrexate, monoclonal antibodies against TNF, IL-12 or IL-23 or a $4 \beta 7$ integrin, the later reducing leukocyte trafficking specifically into the GI tract, are also effective, but do not represent a definitive cure.

\section{Rheumatoid arthritis}

Rheumatoid arthritis (RA), an autoimmune disease with severe joint inflammation, cartilage and bone destruction links strongly to the presence of autoantibodies, such as rheumatoid factor and anti-citrullinated peptide antibodies25. Monocytes, neutrophil, macrophages and dendritic cells promote chronic joint inflammation. Pro-inflammatory cytokines produced by these cells, such as TNF, IL-1 $\beta$, IL-6, IL-12, IL-18 and IL-23, promote the generation of pathogenic $\mathrm{B}$ and $\mathrm{T}$ cells. IFN- $\gamma^{+} \mathrm{IL}-17^{+} \mathrm{CD} 4^{+} \mathrm{T}$ cells mediate cartilage and bone destruction 26 . IL-1 $\beta$ and IL-23 expand these IFN- $\gamma^{+}$IL-17+ IL-22+ Th17 cells 27 . IL-1 $\beta$ and IL-17A induce cartilage and bone destruction, the latter through the RANKL-mediated activation of osteoclasts (Table 1). Anti-TNF monoclonal antibodies or IL-1Ra, anti-IL-6R, anti-IL-12p40 or anti-IL-17A can treat RA26. Small molecule inhibitors of JAK-STAT signalling downstream of inflammatory cytokine receptors have shown efficacy in subsets of patients. 


\section{Atherosclerosis}

Atherosclerosis is characterized by a strong activation of endothelial cells in the inner lining of the arterial lumen, which in turn recruit monocytes via the expression of adhesion molecules. Monocytes mature into macrophages that can replicate 28 and engulf modified lipoprotein particles to form inflammatory foamy macrophages. Excessive deposition of cholesterol in the subintimal space can precipitate cholesterol crystals, which trigger the NLRP3 inflammasome and thus the release of active IL-1 $\beta 29$ (Table 1). Cells of the adaptive immune response including $\mathrm{T}$ and $\mathrm{B}$ cells also enter the artery wall during atherogenesis, where they regulate pathogenic functions of innate immune cells in the plaque30. In clinical practice, biomarkers of inflammation such as high sensitivity C-reactive protein (hsCRP) serve to detect vascular inflammation. Statins (HMG-CoA reductase inhibitors) have proven remarkably successful in preventing first and recurrent cardiovascular events and reduce both cholesterol and hsCRP. Clinical trials are evaluating whether anti-inflammatory agents such as low-dose methotrexate and canakinumab (a monoclonal antibody targeting IL-1 $\beta$ ) can prevent atherosclerotic events.

\section{Neurodegenerative diseases}

Innate immune mechanisms are emerging as a crucial component of normal brain aging, as well as major contributor to neurodegenerative diseases, including but not restricted to Alzheimer's disease (AD), Parkinson's disease (PD) and amyotrophic lateral sclerosis (ALS) 31, 32. Several mutations in genes encoding immune proteins increase the risk to develop AD (TREM2, CD33, PLCG2), ALS (C9orf72, TBK1, CHCHD10) and PD (LRRK2) (Table 1). Activation of microglia in response to beta-sheet structured proteins, misfolded proteins, neuronal debris or aberrant nucleic acids33, triggers neuronal dysfunction, structural damage and ultimately death. Beta-amyloid (A $\beta$ ) fibrils, one of the major pathological hallmarks of AD, induce the activation of the NLRP3 inflammasome and IL- $1 \beta$ generation in microglia through activation of various PRR34. Microglia express receptors for neurotransmitters and neurotrophins, which in turn modulate their function in a brain region-specific manner. Norepinephrine or acetylcholine can suppress excess inflammation in the brain35.

\section{Liver diseases}

Mediators of inflammation, especially cytokines such as IL-1 family cytokines, IL-6 and TNF, control acute liver failure, hepatic acute phase response, steatosis, cholestasis, hypergammaglobulinemia and the development of fibrosis36. In acute injury, hepatic inflammatory cytokines induce necrosis of hepatocytes but also mediate hepatocyte regeneration. Hepatocyte necrosis releases IL-1a, which recruits bone marrow-derived monocytes and macrophages. Macrophages are also rapidly recruited from the peritoneum and they seem to be protective. Fibrosis and the release of TGF- $\beta$ play a crucial role in chronic liver disease. Alcoholic and non-alcoholic fatty liver disease represent sterile inflammation with massive lipid accumulation. Anti-inflammatory strategies have not yet been adequately developed in the management of these disorders. 


\section{Diabetes}

T1D (10\% of cases) is caused by immune-mediated selective $\beta$-cell destruction triggered by unknown environmental factors in individuals with a polygenetic predisposition.

Inflammation plays a crucial role in this process, with a first step in which inflammatory macrophages and proinflammatory cytokines, and in particular IL- $1 \beta$ and TNF, are expressed in the islet cell infiltrate and contribute to $\beta$-cell toxicity. Subsequently, an infiltrate which is lymphocytic in nature develops, with populations of both $\mathrm{CD} 8^{+}$and $\mathrm{CD} 4^{+}$ $\mathrm{T}$ cells displaying an autoreactivity against specific islet antigenic peptides. The $\mathrm{T}$ cells are often accompanied by influent $\mathrm{CD} 20^{+} \mathrm{B}$ cells. Despite promising initial results in small studies, the use of IL-1 or TNF antagonists to improve insulin secretion in new-onset T1D patients has not been successful in phase II trials 37, 38. T2D (90\% of cases) is characterized by defective insulin secretion as well as decreased responses to insulin. IL-1 $\beta$ driven inflammation plays an important role in the $\beta$-cell loss in T2D. Insulin resistance in T2D is also due to liver and fat inflammation through general inflammatory pathways as described above, with IL-1 $\beta$-inflammasome pathway activation playing a crucial role (Table 1). Clinical trials using IL-1Ra and IL-1 $\beta$ antibodies and salsalate 39,40 offer proof of concept of a beneficial effect of dampening inflammation in T2D.

\section{Lung disease}

Innate inflammation and adaptive immunity are essential to lung defense, however, if unchecked, they result in lung disease. Air pollutants with inflammatory effects, including endotoxin, can exacerbate asthma but can also initiate the disease. Whereas IL-4 and DCs promote $\mathrm{T}_{\mathrm{H}} 2$-mediated inflammation, airway epithelia produce IL-1a, IL-1 $\beta$, IL-25, IL-33, and thymic stromal lymphopoetin, each of which recruit and activate ILC2, eosinophils and basophils that enhance inflammation and remodelling of the airway41. IL-4 and IL-13 contribute to the interaction between innate and adaptive immune mechanisms that promote inflammatory airway disease. IL-5 promotes differentiation and activation of eosinophils, and clinical trials that target the IL-5 pathway by blocking the cytokine or its receptor in asthma provide benefit in patients with a high $\mathrm{T}_{\mathrm{H}} 2$ profile.

\section{Chronic kidney disease}

Chronic kidney disease (CKD) is a low-grade inflammatory process. Inflammatory macrophages infiltrate the kidney and induce the release of proinflammatory cytokines and mediators such as IL-1 $\beta$, TNF, IL-6, IL-23, reactive oxygen species, nitric oxide, and iNOS. Cytokines such as TNF or TGF- $\beta 1$ produced locally during kidney inflammation decrease kidney expression of Klotho and PGC-1 $\alpha$ and lead to suboptimal induction of these nephroprotective proteins. Circulating IL-1 $\beta$, IL-1Ra, IL-6 and CRP are elevated in patients with advanced stages of CKD42, predicting a decline in kidney function43. IL-1 $\beta$ contributes to tubular interstitial fibrosis, promotes tubular epithelial-myofibroblast transdifferentiation, interstitial renal fibroblasts, cytokine gene expression, production of prostaglandins E2 by mesangial cells, and TGF- $\beta$-mediated interstitial fibrosis 44 . Treatment with the IL-1 soluble receptor trap (rilonacept) reduced CRP concentrations, improved 
brachial artery flow-mediated dilation, and reduced vascular oxidative stress in patients with CKD45.

\section{Inflammatory skin diseases}

In atopic dermatitis $(\mathrm{AD})$ penetration of external stimuli (e.g. allergens) through an impaired skin barrier leads to an exaggerated $\mathrm{T}_{\mathrm{H}} 2$ response 46 . Local immune imbalance causes further skin barrier deterioration as IL-4 and IL-13 downregulate the expression of major skin barrier genes such as filaggrins, leading to a vicious circle. In psoriasis, a T cell-driven disease with contributions of innate and adaptive immunity, inflammation is driven by signalling through NF- $\mathrm{kB}$ and $\mathrm{T}_{\mathrm{H}} 17$ - and $\mathrm{T}_{\mathrm{H}} 1$-type cytokines, and therapies targeting $\mathrm{TNF}$, IL-12/IL-23 and IL-17 are effective (Table 1). Psoriasis involves systemic inflammatory responses and frequent comorbidities are rheumatological or cardiovascular in nature 47 . In hidradenitis suppurativa, also known as acne inversa, a devastating skin disorder bearing the characteristics of both auto-inflammatory and auto-immune disorders, histopathology reveals heavy lesional deposits of TNF, IL-1 $\beta$, IL-23 and IL-17, as well as activation of both $\mathrm{CD} 4^{+}$and $\mathrm{CD} 8^{+} \mathrm{T}$ cells 48 .

\section{Auto-inflammatory syndromes}

Auto-inflammatory syndromes can be defined as disorders with abnormally increased inflammation, mediated predominantly by the cells and molecules of the innate immune system, with a significant host predisposition. The enhanced inflammatory state encompasses the production of proinflammatory pyrogenic cytokines, especially IL- $1 \beta$, and hence fever and acute phase response are common prominent signs. From a theoretical point of view, auto-inflammatory syndromes result either from excessive production and/or biological activity of inflammatory mediators, or to a lack of endogenous inhibition. The prototype of the latter is DIRA, deficiency of interleukin-1 receptor antagonist, in which a lack of inhibition of IL-1 bioactivity leads to excessive inflammation49. Other important syndromes are familial Mediterranean fever (FMF), cryopyrinopathies, and hyperimmunoglobulinemia D and periodic fever syndrome (HIDS). An exaggerated IL-1 $\beta$ response is the hallmark of many auto-inflammatory disorders, and interference with IL-1 action is the preferred therapy in these disorders.

\section{Cancer}

All the usual components of the inflammatory response reside in the tumour microenvironment but often exhibit 'corrupted' functions. Cancer-related inflammation (CRI) is a key component of the tumour microenvironment 50, 51 and includes inflammatory cells, especially tumor-associated macrophages (TAMs) that affect all aspects of cancer including growth, genetic instability, angiogenesis and metastasis52. TAMs contribute to cancer immunosuppression by producing prostaglandins, products of tryptophan metabolism and expressing triggers of checkpoint blockade (e.g. PD-L1). T and B cells, neutrophils, mast cells and eosinophils are also cellular components of CRI. Inflammatory cytokines such as TNF, IL-6 and IL-1 are important mediators of intercellular communication in CRI, along with many other members of the chemokine family50, 51. The 
humoral arm of innate immunity also participate in CRI. Pentraxin-3 (PTX3), a fluid phase PRR, interacts with complement components and operates downstream of IL-1 in mouse models of carcinogenesis (Table 1). The type of inflammatory reaction dictates the clinical impact of cancer. $\mathrm{T}$ cell-driven inflammation, characterized by an IFN signature, associates with a better prognosis53, whereas high macrophage infiltration generally associates with worse prognosis, especially when considering markers of type 2 polarization52.

\section{Conclusions and future perspectives}

The heterogeneous nature of the inflammatory response depends on the type of disease and organ in which it occurs, and inflammation can have both protective effects, as well as collateral deleterious consequences for the host. The examples of successful therapies that target inflammation underscore the importance of understanding inflammatory pathways to enable further therapeutic advances.

\section{Acknowledgements}

We thank all the colleagues in the field for their contribution to the knowledge in inflammation, and we regret our inability due to space constraints to refer to many important studies that have enlightened important aspects of inflammation.

DLK was supported by the Intramural Research Program of the National Human Genome Research Institute (NHGRI) at the US National Institutes of Health. MGN was supported by an ERC Consolidator Grant (\#310372), a Spinoza Grant of the Netherlands Organization for Scientific Research, and a Competitiveness Operational Programme Grant of the Romanian Ministry of European Funds (FUSE). KLN was supported by the American Heart Association postdoctoral fellowship award 12POST11920023. FC was supported by NIH grants DK042191, DK055812, DK091222, and DK097948. FRB was supported by an ERC Advanced Grant (ERC322566) and a Cancer Research UK Programme Grant (A16354) CAD was supported by an NIH Grant AI15614. LABJ was supported by supported by a Competitiveness Operational Programme grant of the Romanian Ministry of European Funds (HINT, ID P_37_762; MySMIS 103587) and a Dutch Arthritis Foundation grant (NR-12-2-303). KHGM was supported by grants from Science Foundation Ireland. PL was supported by the RRM Charitable Fund, The National Heart, Lung, and Blood Institute (R01 HL080472). BS is supported by the German Research Foundation SPP1656, 749/7-1, 749/10-1, the German Cancer Foundation, the German Israel Foundation and the Horizon 2020 program. DAS was supported by NIH grant R01-HL097163

\section{References}

1. Eltzschig HK, Carmeliet P. Hypoxia and inflammation. N Engl J Med. 2011; 364:656-665. [PubMed: 21323543]

2. Franceschi C, Campisi J. Chronic inflammation (inflammaging) and its potential contribution to age-associated diseases. J Gerontol A Biol Sci Med Sci. 2014; 69(Suppl 1):S4-9. [PubMed: 24833586]

3. Akira S, Uematsu S, Takeuchi O. Pathogen recognition and innate immunity. Cell. 2006; 124:783801. [PubMed: 16497588]

4. Iwasaki A, Medzhitov R. Toll-like receptor control of the adaptive immune responses. Nat Immunol. 2004; 5:987-995. [PubMed: 15454922]

5. Dinarello CA. Historical insights into cytokines. Eur J Immunol. 2007; 37(Suppl 1):S34-45. [PubMed: 17972343]

6. Dinarello CA. Cytokines as mediators in the pathogenesis of septic shock. Curr Top Microbiol Immunol. 1996; 216:133-165. [PubMed: 8791739]

7. Bonecchi R, et al. Chemokines and chemokine receptors: an overview. Front Biosci (Landmark Ed). 2009; 14:540-551. [PubMed: 19273084]

8. Scapini P, Marini O, Tecchio C, Cassatella MA. Human neutrophils in the saga of cellular heterogeneity: insights and open questions. Immunol Rev. 2016; 273:48-60. [PubMed: 27558327] 
9. Eyerich K, Dimartino V, Cavani A. IL-17 and IL-22 in immunity: Driving protection and pathology. Eur J Immunol. 2017; 47:607-614. [PubMed: 28295238]

10. Artis D, Spits H. The biology of innate lymphoid cells. Nature. 2015; 517:293-301. [PubMed: 25592534]

11. Shattuck EC, Muehlenbein MP. Human sickness behavior: Ultimate and proximate explanations. Am J Phys Anthropol. 2015; 157:1-18. [PubMed: 25639499]

12. Ward PA. The harmful role of c5a on innate immunity in sepsis. J Innate Immun. 2010; 2:439-445. [PubMed: 20588003]

13. Ouyang W, Rutz S, Crellin NK, Valdez PA, Hymowitz SG. Regulation and functions of the IL-10 family of cytokines in inflammation and disease. Annu Rev Immunol. 2011; 29:71-109. [PubMed: 21166540]

14. Dinarello CA, et al. Suppression of innate inflammation and immunity by interleukin-37. Eur J Immunol. 2016; 46:1067-1081. [PubMed: 27060871]

15. Dinarello CA. IL-1: discoveries, controversies and future directions. Eur J Immunol. 2010; 40:599_ 606. [PubMed: 20201008]

16. Blom AM. The role of complement inhibitors beyond controlling inflammation. J Intern Med. 2017

17. Joosten LA, et al. Alpha-1-anti-trypsin-Fc fusion protein ameliorates gouty arthritis by reducing release and extracellular processing of IL-1beta and by the induction of endogenous IL-1Ra. Ann Rheum Dis. 2016; 75:1219-1227. [PubMed: 26174021]

18. Andersson U, Tracey KJ. Reflex principles of immunological homeostasis. Annu Rev Immunol. 2012; 30:313-335. [PubMed: 22224768]

19. Hotchkiss RS, Monneret G, Payen D. Sepsis-induced immunosuppression: from cellular dysfunctions to immunotherapy. Nat Rev Immunol. 2013; 13:862-874. [PubMed: 24232462]

20. Singer M, et al. The Third International Consensus Definitions for Sepsis and Septic Shock (Sepsis-3). JAMA. 2016; 315:801-810. [PubMed: 26903338]

21. Takeuchi O, Akira S. Pattern recognition receptors and inflammation. Cell. 2010; 140:805-820. [PubMed: 20303872]

22. Cohen J, et al. Sepsis: a roadmap for future research. Lancet Infect Dis. 2015; 15:581-614. [PubMed: 25932591]

23. van der Poll T, v.d.V F, Scicluna BP, Netea MG. The immunopathology of sepsis and potential therapeutic targets. Nature Rev Immunol. 2017

24. Bamias G, Pizarro TT, Cominelli F. Pathway-based approaches to the treatment of inflammatory bowel disease. Transl Res. 2016; 167:104-115. [PubMed: 26408803]

25. Firestein GS, McInnes IB. Immunopathogenesis of Rheumatoid Arthritis. Immunity. 2017; 46:183-196. [PubMed: 28228278]

26. Englbrecht M, et al. Measuring joint involvement in polyarticular psoriatic arthritis: an introduction of alternatives. Arthritis Care Res (Hoboken). 2010; 62:977-983. [PubMed: 20191568]

27. Sutton C, Brereton C, Keogh B, Mills KH, Lavelle EC. A crucial role for interleukin (IL)-1 in the induction of IL-17-producing T cells that mediate autoimmune encephalomyelitis. J Exp Med. 2006; 203:1685-1691. [PubMed: 16818675]

28. Robbins CS, et al. Local proliferation dominates lesional macrophage accumulation in atherosclerosis. Nat Med. 2013; 19:1166-1172. [PubMed: 23933982]

29. Duewell P, et al. NLRP3 inflammasomes are required for atherogenesis and activated by cholesterol crystals. Nature. 2010; 464:1357-1361. [PubMed: 20428172]

30. Ketelhuth DF, Hansson GK. Adaptive Response of T and B Cells in Atherosclerosis. Circ Res. 2016; 118:668-678. [PubMed: 26892965]

31. Heneka MT, Golenbock DT, Latz E. Innate immunity in Alzheimer's disease. Nat Immunol. 2015; 16:229-236. [PubMed: 25689443]

32. Heneka MT, Kummer MP, Latz E. Innate immune activation in neurodegenerative disease. Nat Rev Immunol. 2014; 14:463-477. [PubMed: 24962261]

33. Kettenmann H, Hanisch UK, Noda M, Verkhratsky A. Physiology of microglia. Physiol Rev. 2011; 91:461-553. [PubMed: 21527731] 
34. Halle A, et al. The NALP3 inflammasome is involved in the innate immune response to amyloidbeta. Nat Immunol. 2008; 9:857-865. [PubMed: 18604209]

35. Pocock JM, Kettenmann H. Neurotransmitter receptors on microglia. Trends Neurosci. 2007; 30:527-535. [PubMed: 17904651]

36. Cressman DE, et al. Liver failure and defective hepatocyte regeneration in interleukin-6-deficient mice. Science. 1996; 274:1379-1383. [PubMed: 8910279]

37. Mastrandrea L, et al. Etanercept treatment in children with new-onset type 1 diabetes: pilot randomized, placebo-controlled, double-blind study. Diabetes Care. 2009; 32:1244-1249. [PubMed: 19366957]

38. Moran A, et al. Interleukin-1 antagonism in type 1 diabetes of recent onset: two multicentre, randomised, double-blind, placebo-controlled trials. Lancet. 2013; 381:1905-1915. [PubMed: 23562090]

39. Larsen CM, et al. Interleukin-1-receptor antagonist in type 2 diabetes mellitus. N Engl J Med. 2007; 356:1517-1526. [PubMed: 17429083]

40. Donath MY. Targeting inflammation in the treatment of type 2 diabetes: time to start. Nat Rev Drug Discov. 2014; 13:465-476. [PubMed: 24854413]

41. Lambrecht BN, Hammad H. The airway epithelium in asthma. Nat Med. 2012; 18:684-692. [PubMed: 22561832]

42. Gupta J, et al. Association between albuminuria, kidney function, and inflammatory biomarker profile in CKD in CRIC. Clin J Am Soc Nephrol. 2012; 7:1938-1946. [PubMed: 23024164]

43. Tonelli M, et al. Biomarkers of inflammation and progression of chronic kidney disease. Kidney Int. 2005; 68:237-245. [PubMed: 15954913]

44. Burns KD. Interleukin-1beta as a mediator of tubulointerstitial fibrosis. Kidney Int. 2002; 62:346347. [PubMed: 12081597]

45. Nowak KL, et al. IL-1 Inhibition and Vascular Function in CKD. J Am Soc Nephrol. 2017; 28:971-980. [PubMed: 27647856]

46. Palmer CN, et al. Common loss-of-function variants of the epidermal barrier protein filaggrin are a major predisposing factor for atopic dermatitis. Nat Genet. 2006; 38:441-446. [PubMed: 16550169]

47. Noda S, Krueger JG, Guttman-Yassky E. The translational revolution and use of biologics in patients with inflammatory skin diseases. J Allergy Clin Immunol. 2015; 135:324-336. [PubMed: 25541257]

48. Kanni T, et al. Compartmentalized Cytokine Responses in Hidradenitis Suppurativa. PLoS One. 2015; 10:e0130522. [PubMed: 26091259]

49. Aksentijevich I, et al. An autoinflammatory disease with deficiency of the interleukin-1-receptor antagonist. N Engl J Med. 2009; 360:2426-2437. [PubMed: 19494218]

50. Balkwill F, Mantovani A. Inflammation and cancer: back to Virchow? Lancet. 2001; 357:539-545. [PubMed: 11229684]

51. Mantovani A, Allavena P, Sica A, Balkwill F. Cancer-related inflammation. Nature. 2008; 454:436-444. [PubMed: 18650914]

52. Mantovani A, Marchesi F, Malesci A, Laghi L, Allavena P. Tumour-associated macrophages as treatment targets in oncology. Nat Rev Clin Oncol. 2017

53. Gajewski TF, Schreiber H, Fu YX. Innate and adaptive immune cells in the tumor microenvironment. Nat Immunol. 2013; 14:1014-1022. [PubMed: 24048123] 


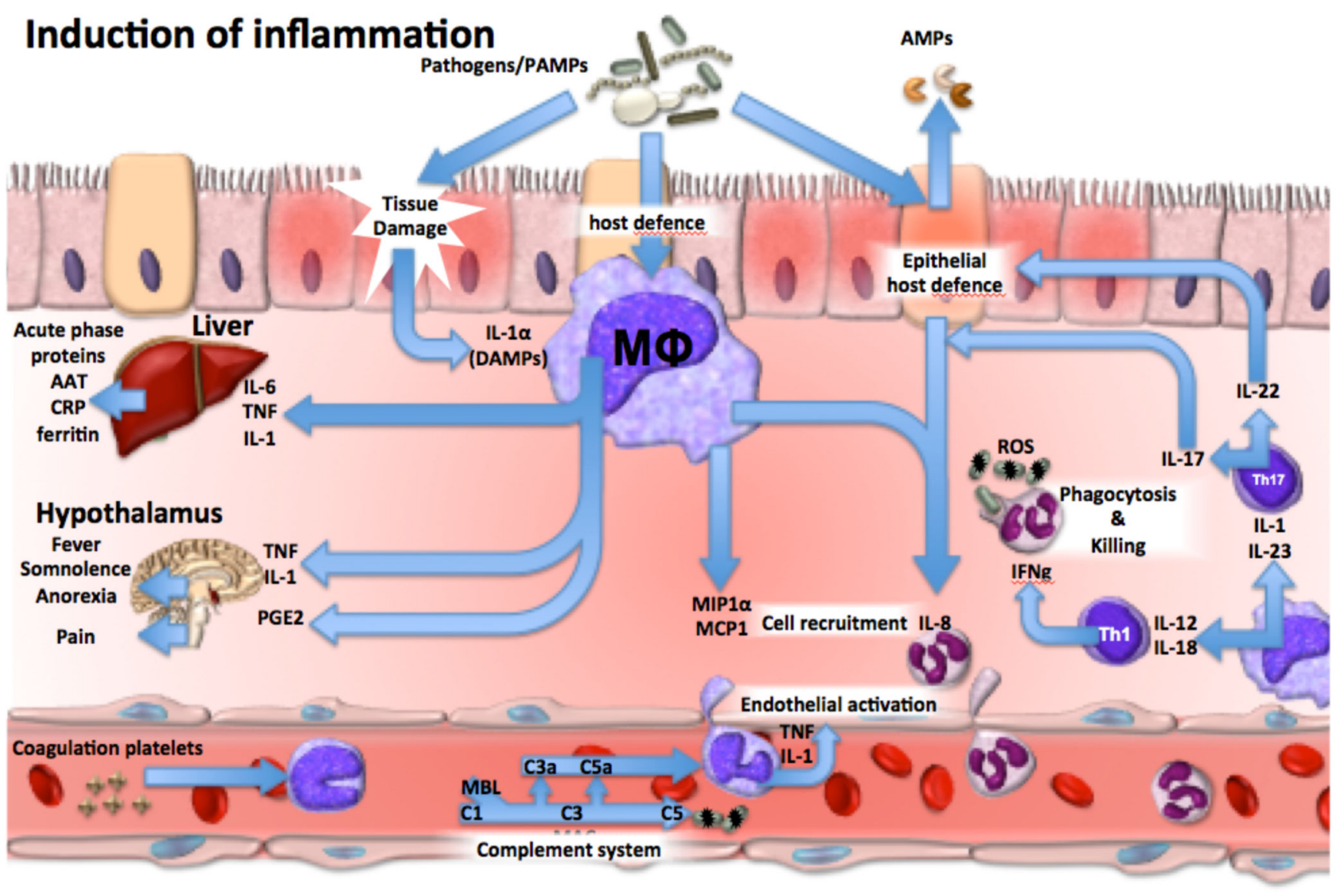

Figure 1.

The immunological mechanisms leading to the induction of inflammation during the first stages of host defense against invading pathogens. a 1-antitrypsin (AAT), pathogen associated molecular patterns (PAMPs), antimicrobial peptides (AMPs), danger associated molecular patterns (DAMPs), membrane attack complex (MAC), reactive oxygen species (ROS), C-reactive protein (CRP), tumour necrosis factor (TNF), interferon- $\gamma($ IFN- $\gamma$ ), mannose binding lectin (MBL), macrophage or monocyte (M $\phi), \mathrm{C}-\mathrm{C}$ Motif Chemokine Ligand (CCL). 


\section{Resolution of inflammation}

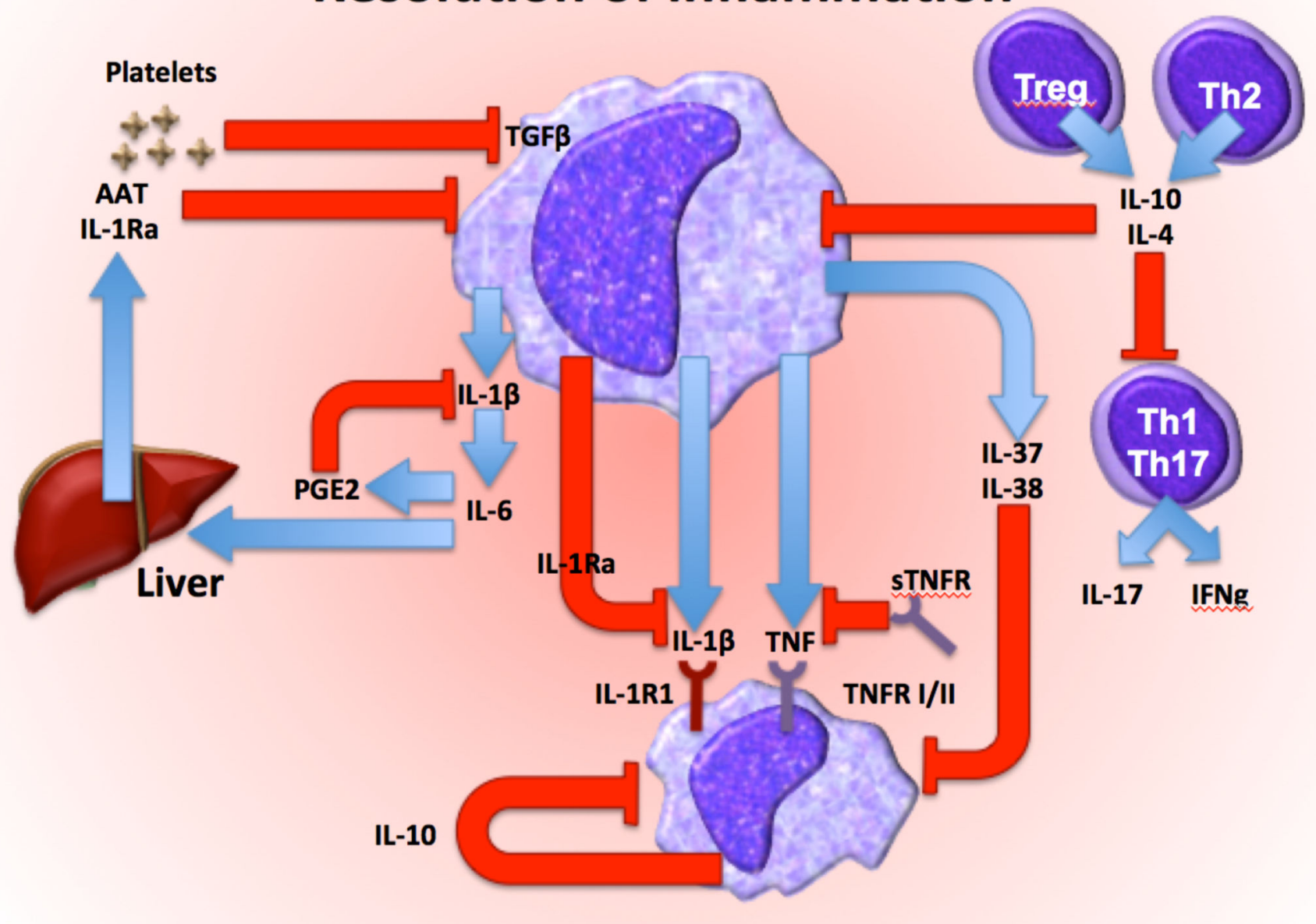

Figure 2.

The regulatory mechanisms that modulate inflammation, leading to resolution after the invading pathogens have been eliminated. Transforming growth factor $\beta$ (TGF- $\beta$ ), a 1antitrypsin (AAT), interleukin-1 receptor antagonist (IL-1Ra), interleukin-1 receptor type I (IL-1R1), interleukin-1 receptor type II (IL-1R2) tumour necrosis factor (TNF), soluble TNF receptor (sTNFR), TNF receptor type I/II (TNFR I/II), prostaglandin E2( $\left.\mathrm{PGE}_{2}\right)$, interferon$\gamma($ IFN- $\gamma)$, macrophage or monocyte $(\mathrm{M} \phi)$ 


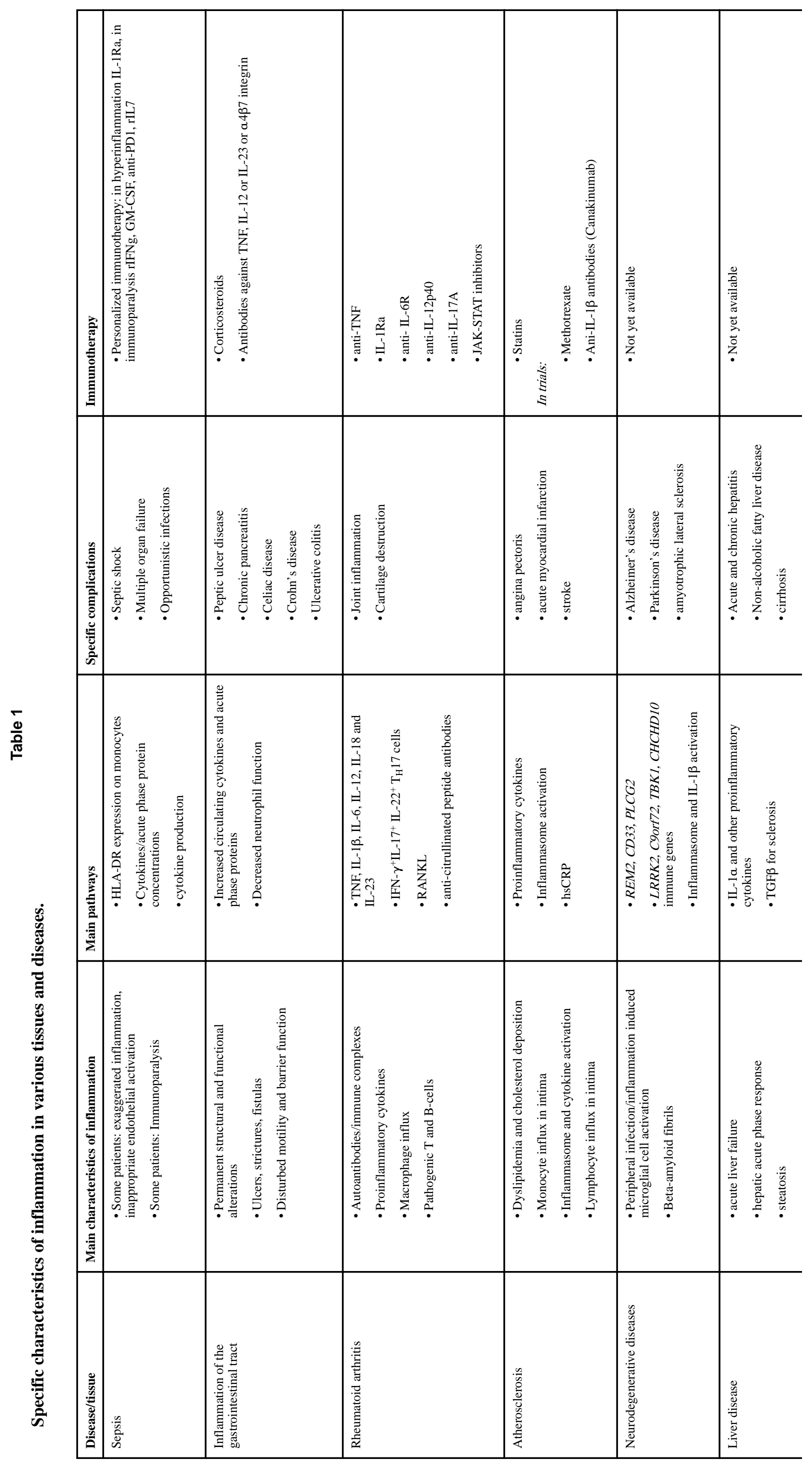

Nat Immunol. Author manuscript; available in PMC 2018 May 08. 


\begin{tabular}{|c|c|c|c|c|c|c|}
\hline 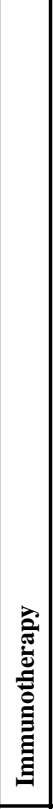 & 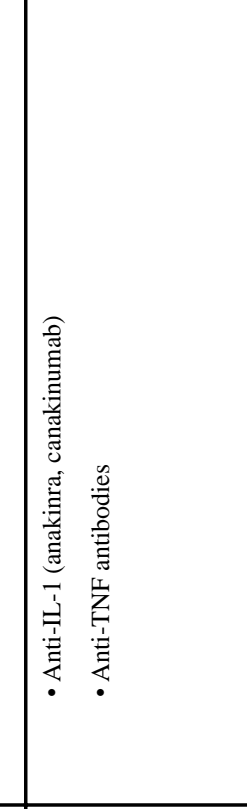 & 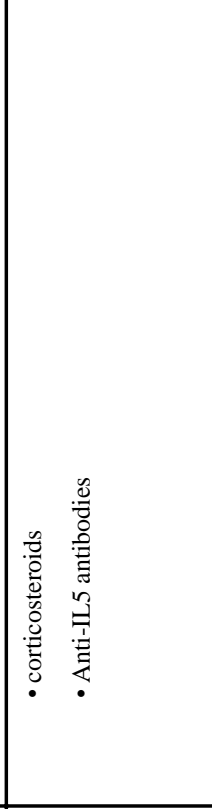 & 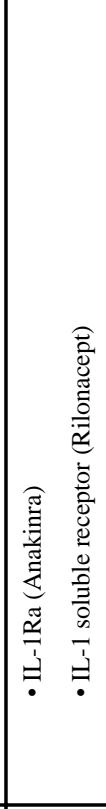 & 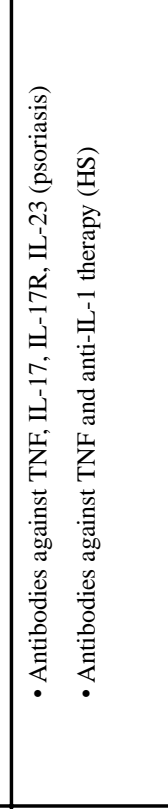 & 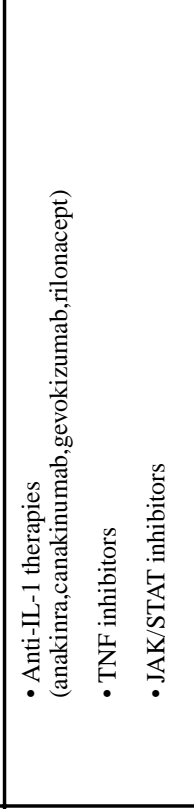 & 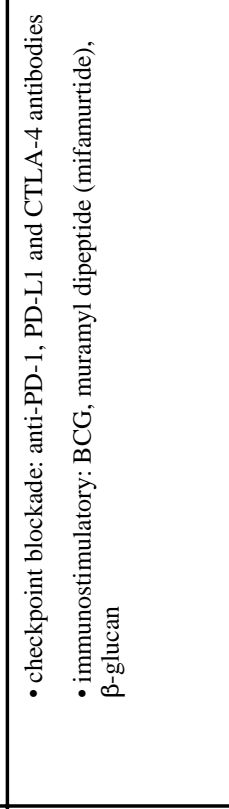 \\
\hline 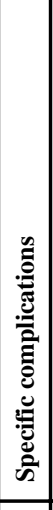 & 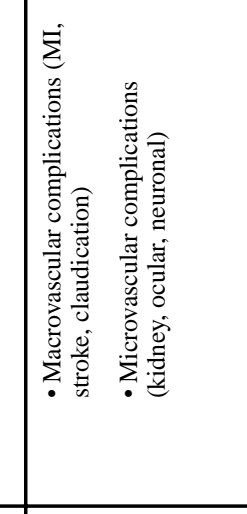 & 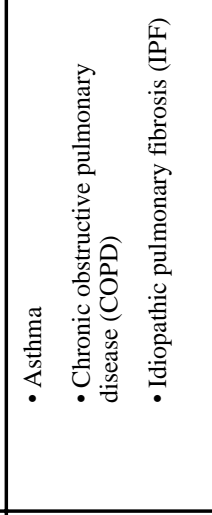 & 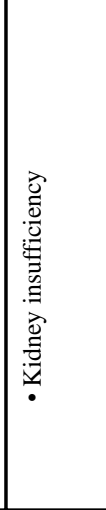 & 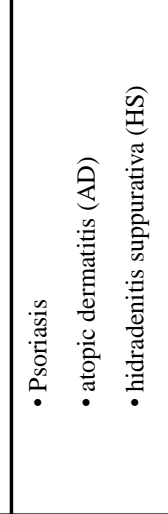 & 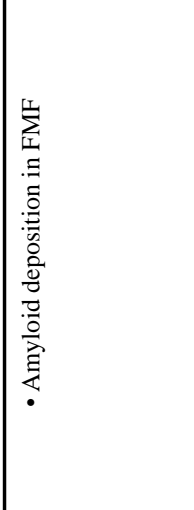 & 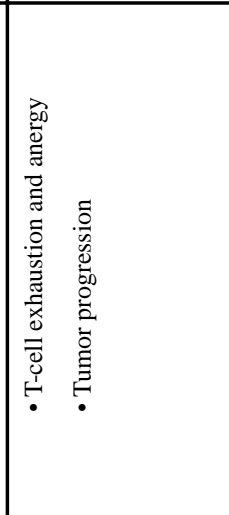 \\
\hline 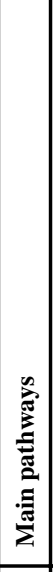 & 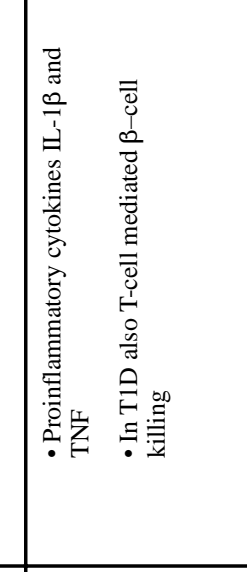 & 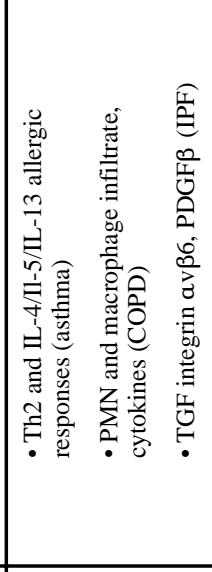 & 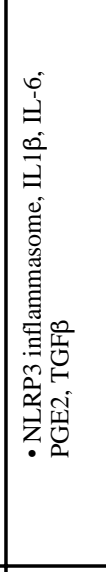 & 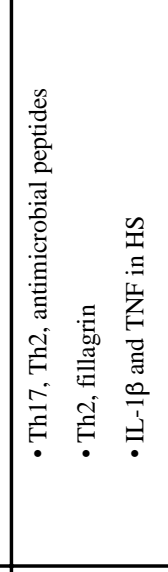 & 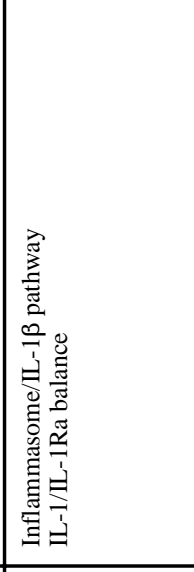 & 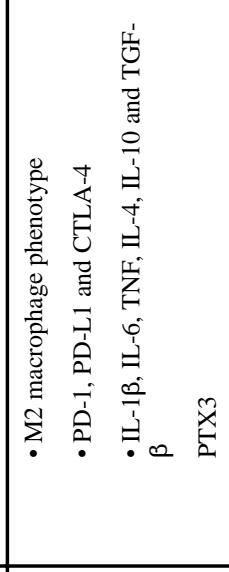 \\
\hline 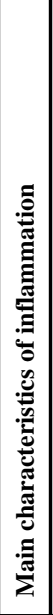 & 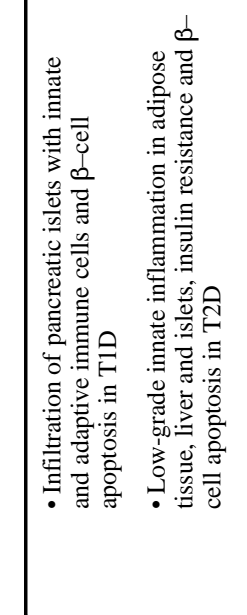 & 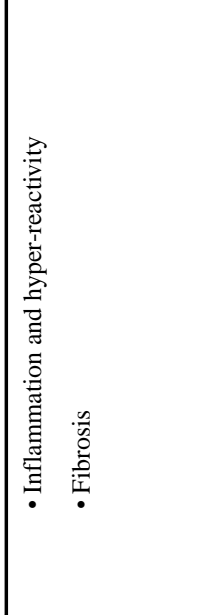 & 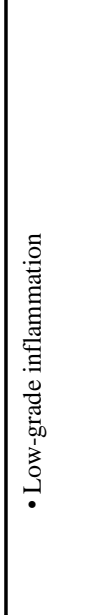 & 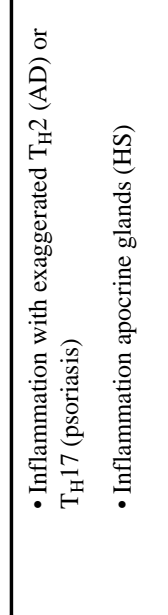 & 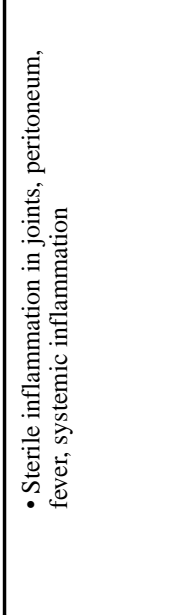 & 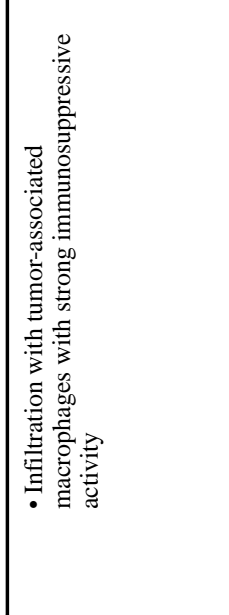 \\
\hline 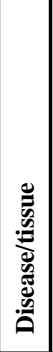 & 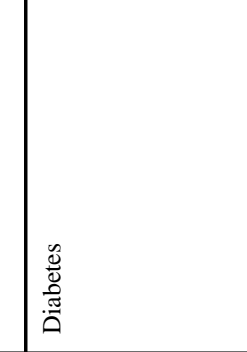 & 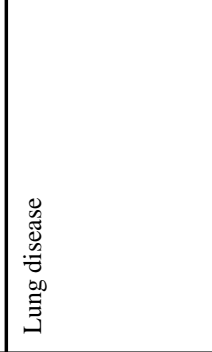 & 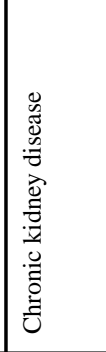 & 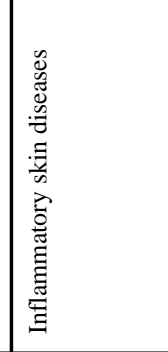 & 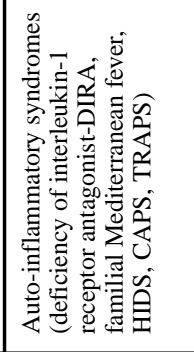 & 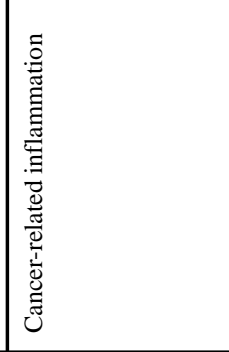 \\
\hline
\end{tabular}

